\title{
PROPAGATION AND ABSORPTION OF ELECTRON CYCLOTRON MASIR EMISSION DURING SOLAR FLARES
}

\author{
M. E. Mckean, R. M. Winglee, and L. A. Dulk \\ Department of Astrophysical, Planetary and Almospheric Sciences, University of \\ Coloralo, Boulder, CO 80909-0391.
}

\begin{abstract}
The propagation and absorption of the maser radiation emitted during solar flares are examined through linear theory and electromagnetic particle sinmlations. While linear theory predicts complete absorption of the maser radiation at the second harmonic layer (where the local cyclotron frequency is roughly one-half the frequency of the maser radiation), particle simulations indicate that some radiation escapes clue to reemission of absorbed energy by the anbient plasma. Strong perpendicular lecating of the ambient electrons occurs as well.
\end{abstract}

\section{INTRODUCTION}

Microwave spike bursts in solar and stellar flares are attributed to the electron cyclotron maser (ECM) instability (Holman et al. 1980); Melrose and 1) ulk 198.2., 198.1; Sharma et al. 1982; Bastian and Bookbinder 1987), which produces intense, highly circularly polarized radiation at the fundamental and harmonics of the local gyrofrepuency $\Omega_{e}$.

One of the fundamental problems associated with altributing microwave spike bursits to the ECM instability is determining how the maser radiation escapes from the solar corona. Specifically, as the radiation propagates outwards, it encounters a second harmonic: absorption liver, where the magnetic held magnitude has about one-half the value it hats at the point of emission and where strong, gyroresonant absorption can occur. Calculations of the optical depth in the second harmonic absorption layer for nearly perpendicular propagation have shown that it is several orders of magnitude greater than unity (Melrese and Dulk 1982a, b, 1984), i.e., the radiation cannot get through.

The purpuse of this investigation is to explore in detail: i) how and how much the incident maser radiation escapes from the second harmonic layer to produce the observed microwave spike bursts; and ii) how the absorbed radiation heats the plasma. Rosults of calculations using linear theory indicate that the linear response of the ambient plastnat to the incident maser radiation is complete absorption of the radiation under virtually all circumstances. Iti order to investigate the nonlinear response of the plasma to the radiation, 1-D electromagnetic particle simulations are used. The code employed for these simulations is similar to the code used by Winglee et al. (1988).

\section{SIMULATION RESUITS}

The simulation model used is illustrated in Fig. 1, along with the magnetic field profile. An energtic population of electrons with an unstable DGII distribution is locited between $x_{3}$ and $x_{1}$ and is responsible for the initial emission of maser radiation. $\Lambda$ cool, Naxwellian population of electrons to the lelt of the energetic population represents the second liarmonic ambient plasma. Another population of cool electrons to the right of the enegetic electrons serves to reflect radiation propagatin:s to the ight due to the increased magnetic feld there. Plasma parameters associatcd with cacl electron population are indicated. The density of the electrons is a constant function of $x$. lons are treated as 
infinitely massive, fixed particles. The magnetic field lies perpendicular to the dimension of the simulation, so that only radiation propagating at right angles to the magnetic field iis investigated. The physical size of the simulation system is several tens of meters for typical solur parameters. The optical depth from linear theory for the second harmonic lityer in the simulation is about 4.

T'wo simulations were performed: an undriven case, in which the initial distribution of cnergetic electrons was allowed to relax, and a driven case, in which the unstable cuergetic clectron distribution was maintained through a process known as "recycling".

Fig. 2 illustrates the alssorption of the largest pulse (labeled "C") emitted by the energetic plasma in the undriven simulation. This figure shows the Poynting flux as a function of position for four different times. Negative values of $S(x)$ indicate radiation propargating to the left; positive values, to the right. Pulse $\mathrm{C}$ is emerging from the energetic plasma in (ia), and undergoes heavy absorption as it propagates through the the absorbing plisma ((b) and (c)). On the other hand, a trailing pulse (labeled "c") undergoes no absorption, and actually grows in energy as it propagates through the absorbtion layer phisma. ((c) and (d)). 'This growth is due to recmission of absorbed energy by the plasma leated by pulse C. This reemission may be an example of "triggered" emission, as discussed by Winglee (1985) and references therein. About $20 \%$ of the incident energy escapes the siecond harmonic layer in these simulations due to reemission processes.

In the driven simulation, the second harmonic plasma is constantly bombarded with pulses of maser radiation and undergoes continuous heating due to the absorption of this radiation. The nature and extent of this heating is shown in Fig. 3, which shows 4 snitpshots of $x-u_{x}$ phase space for the driven case, where $u_{x}=p_{x} / m_{e}$ and $p_{x}$ is is component of the perpendicular momentum. Fig. (a) shows the initial phase space configuration. In (b), a heating edge which marks the right side of the second harmonic lityer has developed near $x / \Delta=1100$. Most of the absorption (and therefore most of the heating) occurs in a small region behind this edge, which corresponds to around $10 \mathrm{~m}$ in the solar corona. By the end of the simulation (d), the electrons in the region immediately behind the heating edge have undergone a $200 \%$ increase in perpendicular energy. The time scale for this increase is about $1 \mu \mathrm{sec}$ for an emission frequency of $1 \mathrm{GHz}$. Thus, absorption of maser radiation can cause significant perpendicular heating of electrons on short lime and length scales.

'This work was supported by NASA's Solar Terrestrial Theory and Solar Ileliospheric Program under grant NAGW-91 and NSG-7287 and by NSF's Solar-Terrestrial Division Hrant A'TM 87-19371 to the University of Colorado, Boulder. The computer simulations were performed on the CRAY X-MP at the San Diego Supercomputer Center which is supported by the Nat onal Science Foundation.

\section{REFERENCES}

Batstian, 'T. S. and Bookbinder, J. A.: 1987, Nature, 320, 678.

llolman, G. D., Eichler, D., and Kundu, M. R.: 1980, in Radio Physics of the Sun, Proc, IAU Symp No. 86, eds. M. R. Kundu and T. E. Gergely, Dordrecht: Reidel, p. 457.

Melrose, D. B. and Dulk, G. A.: 1982a, Astrophys. J., 259, 844.

Melrose, D. B. and Dulk, G. A.: 1982b, Astrophys. J. (Letters), 259, L41.

Mulrose, D. 13. and Dulk, G. A.: 1984, Astrophys. J., 282, 308.

Sharma, R. R. and Vlahos, L.: 1983, Astrophys. J. (Letters), 273, L95.

Winglee, R. W.: 1985, J. Ceophys. Res., 00, 5141.

Winglee, R. W., Dulk, G. A., and Pritchett, P. L.: 1988, Astrophys. J., 328, 809. 


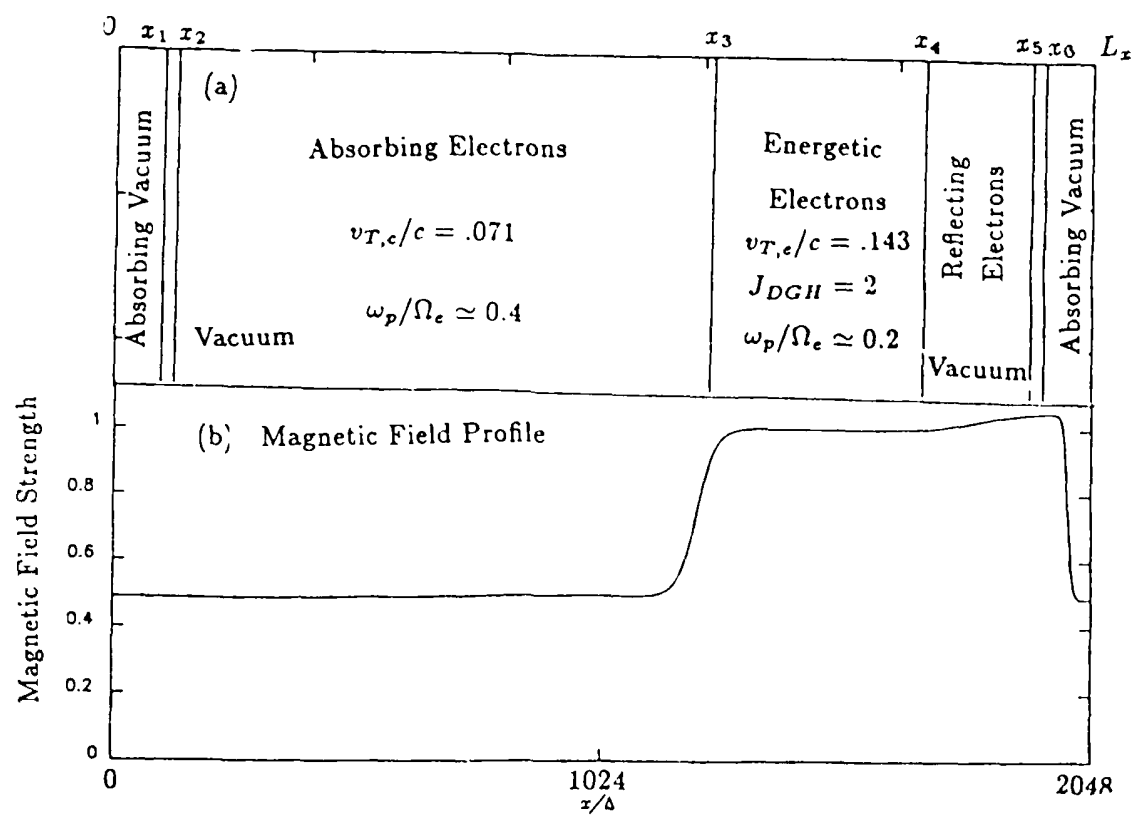

Fig. 1: The simulation model and the magnetic field profile. The magnetic field is perpendicular to the $x$-axis. The thermal velocity $\left(v_{T}\right)$ and ratio of plasma frequency to gyrofrequency $\left(\omega_{p} / \Omega_{e}\right)$ for the energetic and absorbing electron populations are shown.
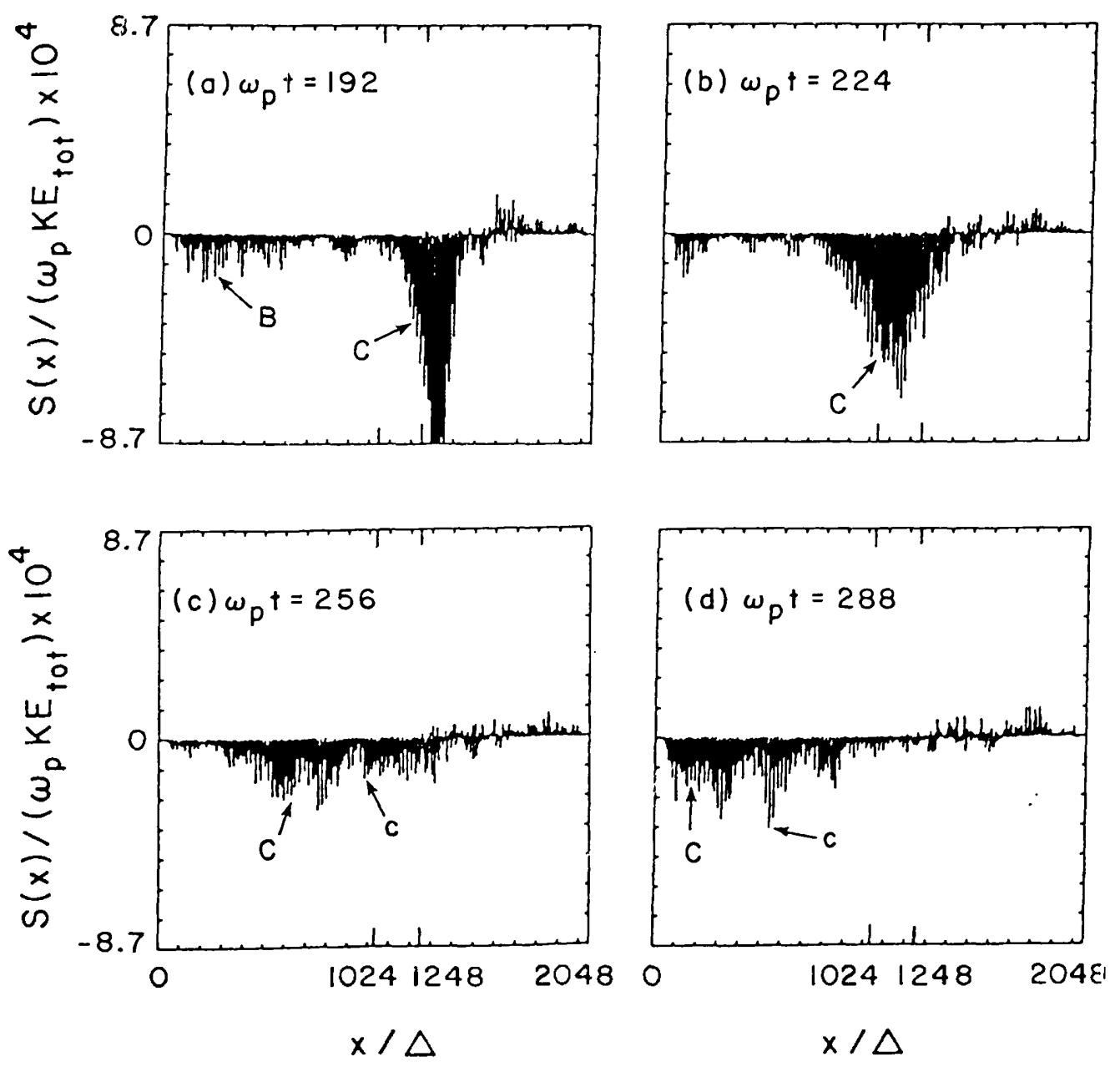

Fig. 2: Snapshots of the Poynting flux $S(x)$ at four different times depicting the absorption of pulse $C$ and a reemission event (pulse $c$ ). The absorbing plasma lies to the right of $x / \Delta=1248$. 

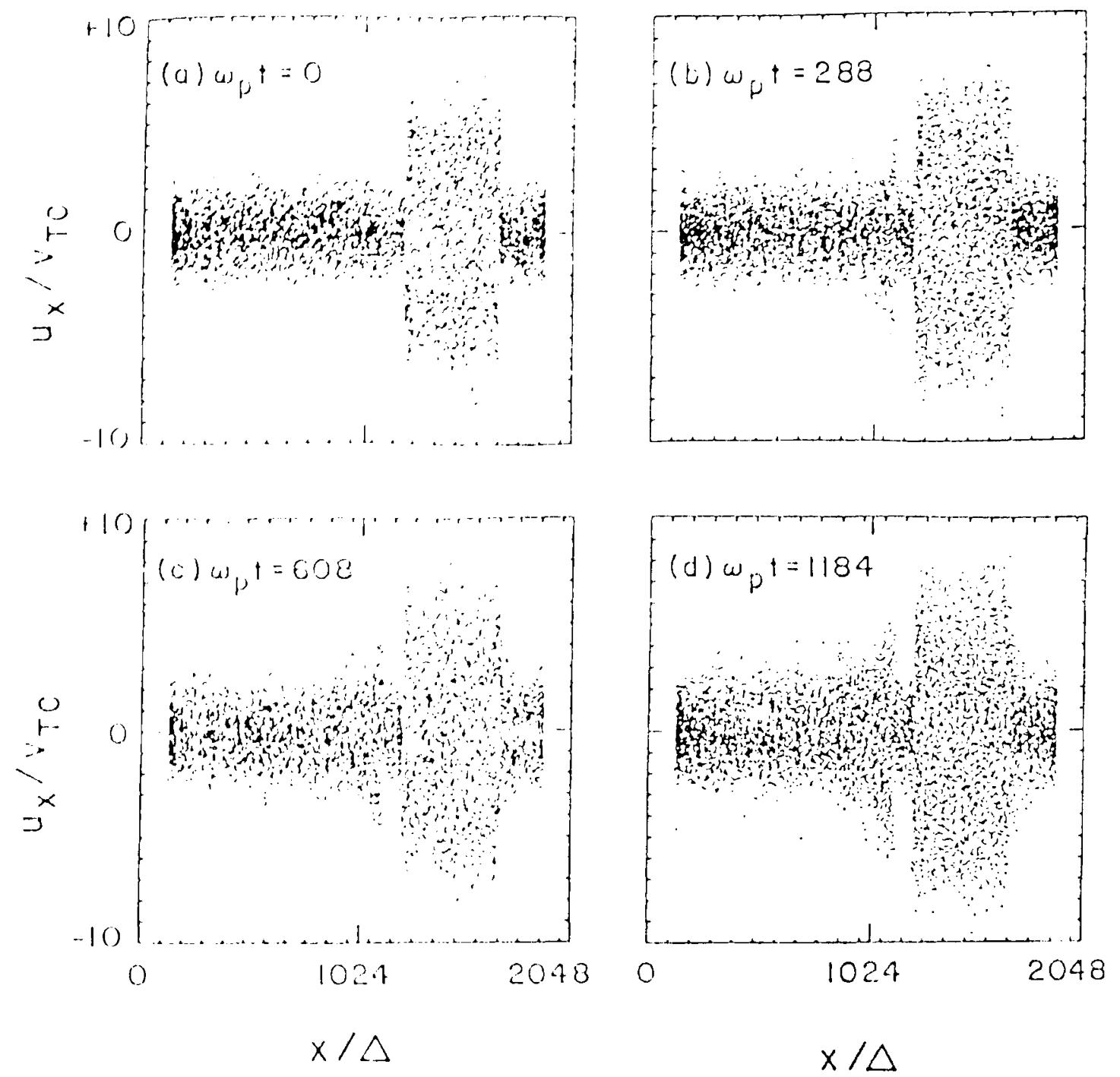

Fig. 3: "lhe hating of the alsorbing plasma by maser radiation is depicted for the driven cate in these snaphihuts of $x-u_{x}$ phase space. 